\title{
Airflow and Aerosol-Drug Delivery in a CT Scan Based Human Respiratory Tract with Tumor Using CFD
}

\author{
V. K. Srivastav, A. Kumar, S. K. Shukla, A. R. Paul†, A. D. Bhatt and A. Jain \\ Motilal Nehru National Institute of Technology Allahabad, Allahabad-211004, India \\ $\dagger$ Corresponding Author Email: arpaul2k@yahoo.co.in
}

(Received March 20, 2013; accepted May 16, 2013)

\begin{abstract}
This paper is focused on to study the effect of a tumor present in the respiratory tract (in trachea) on airflow pattern and aerosol-drug deposition. A realistic model of human respiratory tract was constructed from spiral computed tomography (CT) scan data and a bifocal tumor (Glomus tumor) was constructed in the tract. The inspiratory flow characteristics of the realistic human airway models (with and without tumor) was numerically solved using the realizable $k-\varepsilon$ turbulence model for airflow and Shear Stress Transport (SST) $k-\omega$ turbulence model for two-phase flow. The velocity (contours and vector plots), wall shear stress and deposition efficiency of aerosol were obtained at different locations to the upstream and downstream region of the bifocal tumor in respiratory tract. The flow pattern shows that the maximum flow disturbance occurs around the tumor and at downstream of the flow. Magnitude and location of maximum wall shear stress in the presence of the tumor helps in identifying the extent and probable location of the wall injury during the normal and heavy breathing conditions. Deposition efficiency of aerosol-drug on tumor location will be useful for designing the efficient targeted drug delivery system.
\end{abstract}

Keywords: Human respiratory tract, Glomus tumor, Wall shear stress, Computational fluid dynamics (CFD), Aerosoldrug delivery.

\section{INTRODUCTION}

Application of computational fluid dynamics (CFD) to study the airflow in human respiratory tract is one of the emerging fields of biomedical engineering to assist the medical practitioners in diagnosis and prognosis of the various respiratory diseases. Uncontrolled growth of cells in the lower respiratory tract that eventually forms a mass is known as lung tumor. Segal et al.(2000) mentioned that benign tumors(non-malignant) are found in larynx, trachea and main bronchi. A recent study conducted by American Cancer Society reported that about 222520 new cases and 157300 deaths are reported in USA due to lung tumor in the year 2010 (Case Report, 2010). The airflow fields are much affected by the tumors that create the obstruction in the airflow path in the respiratory tract.

Martonen and Guan(2010) discussed effects of various locations and sizes of tumors on pharmacologic drugs. They simulated airflow pattern in respiratory tract with tumor on carina using a CFD package FIDAP. Human respiratory tract model proposed by Weibel (1963) was used for CFD simulation in this case. The tumor size was defined by $r / R$, where, $r$ is the tumor radius and $R$ is the radius of the airway. It was found that the tumor had foremost effect when $r / R \geq 0.8$ for third and fourth bifurcations and when $r / R \geq 0.6$ for seventh and eighth bifurcations of the respiratory tract. An effect of tumor size and ventilator parameters on localized flow pattern was also reported in the study. They observed that the size of tumor and inhalation flow rates has prominent effect on the flow pattern. Kleinstreuer and Zhang (2003) studied the hemispherical tumors in the fourth generation respiratory tract model. Effects of hemispherical tumors of different sizes and locations were considered in their study. Third to sixth generation of the respiratory tract model was constructed from Weibel(1963). They found that most of the particles deposited on the tumor surface and the tumor not only affected the downstream flow fields but to a small extent upstream flow fields too. Martonen and Guan(2001) studied the drug particle motion in bifurcated human respiratory tract with tumor. They found that an increase in particle size, breathing intensities and tumor size influence drug deposition on the tumor. The computation was performed using CFD package FIDAP. They found that the particles are likely to be deposited on the tumor. Segal et al.(2000) discussed the affect of tumor size and location in the respiratory tract of a four year old child. It was observed that the tumors have a prominent effect on both, the 
localized velocity profiles in respiratory tract as well as in the bulk flow distribution within the lung. Particle deposition in obstructed respiratory tract was presented by Luo et al.(2007). The fourth generation model was based on Weibel model(1963). They obtained significant effects on the particle deposition in downstream of the obstruction. Micron size particle distribution in human upper respiratory tract based on regular pipes was studied by Huang and Zhang (2011). They mentioned inertial impaction is the main mechanism for deposition of particles in both oropharynx and primal generation of respiratory tract. It is reported that wall shear stress value above $0.4 \mathrm{~N} / \mathrm{m}^{2}$ will increase the susceptibility of damage of inner lining of cell in respiratory tract (Lin et al. 2007; Green 2004; Sulaiman 2008). Also wall shear stress above $0.7 \mathrm{~N} / \mathrm{m}^{2}$ will certainly damage the respiratory wall (Koombua and Pidaparti 2008).

On the other side, Watanabe et al.(1998) studied the glomus tumor, which was found in the lower part of trachea. Glomus tumor is normally found as benign tumor. It is soft tissue tumor which is highly vascularised and seems like modified cells of glomus body (Zoltan and Zhang 2010). These are generally solitary in nature but multiple tumors may also exist. It is composed of three components: (i) round or ovoid glomus cells. (ii) vasculature or blood supply cells (iii) smooth cells. The dimensions of different glomus tumor (in trachea) studied by the researchers are listed in Table 1 .

Table 1 Dimensions of Different Glomus Tumor

\begin{tabular}{|l|c|c|}
\hline Author's & $\begin{array}{c}\text { Dimension, } \\
\mathrm{cm}^{3}\end{array}$ & $\begin{array}{c}\text { Shape } \\
\text { (glomus } \\
\text { tumor) }\end{array}$ \\
\hline Watanabe et al.(1998) & $2.0 \times 1.6 \times 1.4$ & Polypoid \\
\hline Colaut et al.(2008) & $2.0 \times 1.0 \times 1.0$ & Polypoid \\
\hline Parker et al.(2010) & $2.0 \times 1.6 \times 1.5$ & Polypoid \\
\hline Shang et al. $(2010)$ & $\begin{array}{c}\text { Case-1: } \\
\end{array}$ & Polypoid \\
\cline { 2 - 3 } & $\begin{array}{c}\text { Case- } 2: \\
1.8 \times 1.5 \times 1.4\end{array}$ & Polypoid \\
\hline
\end{tabular}

All these investigators have discussed the effect of tumor in human respiratory tract based on simplified Weibel model (1963). Srivastav and Jain (2011) used realistic (CT scan) respiratory model for the study of wall shear stress during the heavy breathing condition. Hence it can be inference from the above discussion that there is practically no literature available in which realistic human respiratory tract model was used for the study of the effects of glomus tumor on the airflow patterns. The novelty of the present study is it has addressed this issue. The present paper discusses the effect of glomus tumor in a realistic human respiratory tract using computational fluid dynamics. Computational results, viz. velocity, wall shear stress and drug deposition efficiency furnished in the study will be useful for diagnosis, treatment and prognosis.

\section{GEOMETRY OF HUMAN RESPIRATORY TRACTS}

The geometry of the human respiratory tract was obtained from CT data with the slice increment of $3 \mathrm{~mm}$ and pixel size of $512 \times 512$. The CT scan was started from the inlet of trachea and extended up to the lungs. In this study, total 38 slices were considered for construction from the inlet of trachea to the main bronchus of the lungs. The human respiratory tract model is given in Fig.1.

\subsection{Respiratory Tract without Tumor}

The geometry of the realistic respiratory tract model without tumor constructed from the CT scan data using MIMICS software is shown in Fig.1(a), which consists of the trachea and first generation bronchus having an inlet cross-sectional area of the trachea $(A)$ of $2.73 \mathrm{~cm}^{2}$ and perimeter of the inlet $(P)$ of $6 \mathrm{~cm}$ and hydraulic diameter $(D=4 A / P)$ of $1.82 \mathrm{~cm}$.

\subsection{Respiratory Tract with Tumor}

The tumor in the trachea was adopted from the case reported by Watanabe et al.(1998). They have studied the lower tracheal glomus tumor of a 43 year old patient. The size of the tumor was $2.0 \times 1.6 \times 1.4 \mathrm{~cm}^{3}$. Bronchoscope was used to visualise the polypoid tumor, which is shown in Fig.2. Similar tumor was created in a respiratory tract model at the $2 \mathrm{~cm}$ distance above the carina on the posterior wall (i.e., directly behind) of trachea as shown in Fig.1(b) and Fig. 3. 


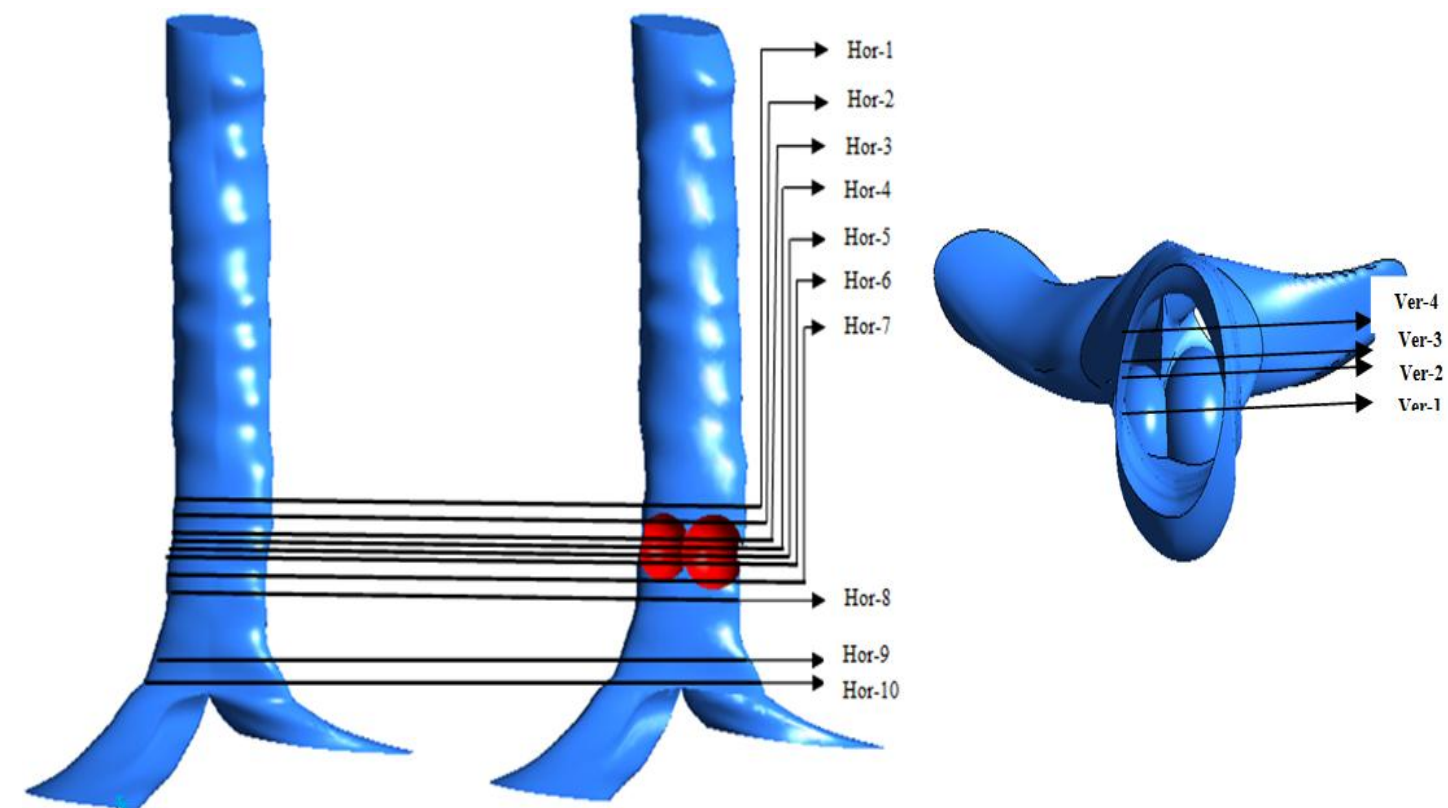

(1a) Respiratory tract without tumor

(1b) Respiratory tract with tumor

Fig. 1. Human Respiratory Tract

Total ten horizontal (Hor-1 to 10) and four vertical (Ver1 to 4) cross-sections (Fig.1) are created for the data analysis in and around the tumor. Hor-1 and Hor- $8,9,10$
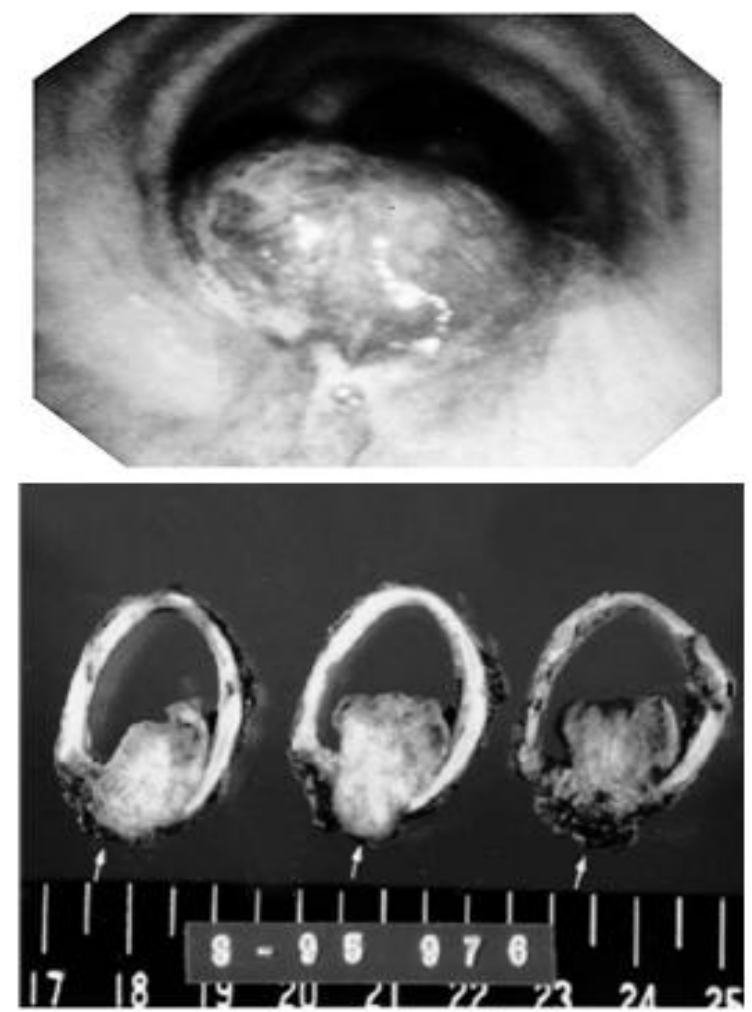

Fig. 2. Glomus tumor in the membranous portion of trachea just above the carina, occupying half of the lumen. Source: Watanabe et al. (1998). Japanese Journal of Surgery. are located at the upstream and downstream respectively. However, Hor-2 to Hor-7 is placed on the tumor.

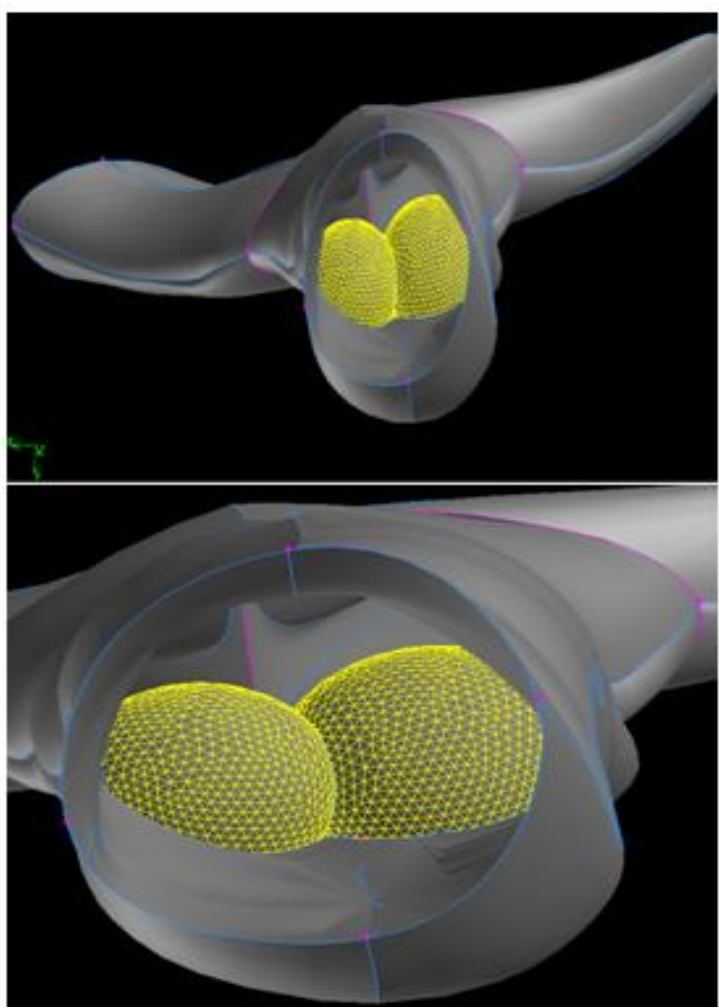

Fig. 3. Glomus tumor constructed for CFD simulation. Present realistic model with polypoid tumor of dimension $2.0 \times 1.6 \times 1.4 \mathrm{~cm}^{3}$ (approx). 


\section{COMPUTATIONAL GRID GENERATION}

Due to the irregular and asymmetrical shape of human respiratory tract, a combination of triangular (surface mesh), tetrahedral and polyhedral elements (volume mesh) were used to improve the quality of mesh. The grid independency test was performed for the different size of elements. The total computational numbers of elements generated after the grid generation in two cases (without tumor and with tumor) are given in Table 2. The grid independency test for respiratory tract with tumor for two phase flow is shown in Fig.4.

Table 2 Computational Grids with Number of Grid Independent Elements

\begin{tabular}{|l|l|l|}
\hline $\begin{array}{l}\text { Human Respiratory } \\
\text { (Cases) }\end{array}$ & $\begin{array}{l}\text { Number of } \\
\text { Elements }\end{array}$ & $\begin{array}{l}\text { Types of } \\
\text { elements }\end{array}$ \\
\hline Without Tumor (Air) & 580037 & Tetrahedral \\
\hline $\begin{array}{l}\text { With Tumor (Air and } \\
\text { Aerosol) }\end{array}$ & 673837 & $\begin{array}{l}\text { Tetrahedral } \\
\text { and } \\
\text { polyhedral }\end{array}$ \\
\hline
\end{tabular}

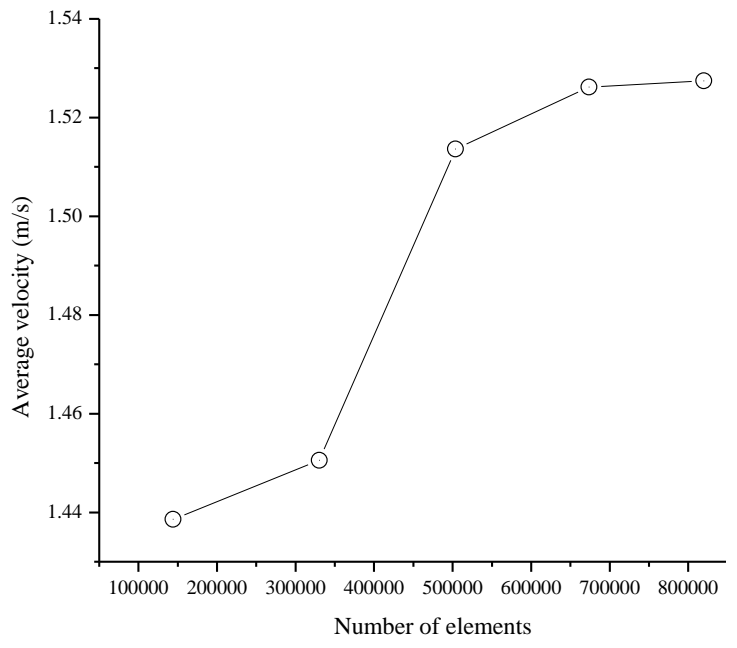

Fig. 4. Grid Independency Test

\section{BOUNDARY CONDITIONS}

The boundary conditions used in this study are velocity at the inlet, zero gauge pressure at the outlet and fixed wall with no slip. In addition, wall of the respiratory tract was assumed rigid. The properties of air at normal conditions were considered (i.e., density is $1.2 \mathrm{~kg} / \mathrm{m}^{3}$ and dynamic viscosity is $1.8 \times 10^{-5} \mathrm{Ns} / \mathrm{m}^{2}$ ). Inspiratory flow rate of 6 and 45 litre per minute for single phase, which corresponds to normal and heavy breathing rate were considered for the study. Boundary conditions applied for both the cases, i.e. without tumor and with tumor remain unchanged.

The aerosol-drugs form droplet having density correspondent to water droplet and is applied in this study. The diameter of aerosol-particle is taken as 1,5 , 7.5 and 10 micron whereas density is considered as 1000 $\mathrm{kg} / \mathrm{m}^{3}$. Three different inspiratory flow rates $(20,45$ and 60 litre/min) were used for discrete phase to bring maximum drug deposition on tumor. Escape option available in Ansys-Fluent was enabled at the inlettrachea and outlets while trap condition at the walls.

\section{GOVERNING EQUATIONS}

The governing equations for the airflow in the respiratory tract with and without tumor are given as follows:

Continuity equation:

Since flow has assumed to be steady and incompressible therefore,

$$
\frac{\partial u_{i}}{\partial x_{i}}=0
$$

Momentum equations:

$u_{j} \frac{\partial u_{i}}{\partial x_{j}}=-\frac{1}{\rho} \frac{\partial p}{\partial x_{i}}+\frac{\mu}{\rho} \frac{\partial}{\partial x_{j}}\left(\frac{\partial u_{i}}{\partial x_{j}}+\frac{\partial u_{j}}{\partial x_{i}}\right)$

$\mu=$ dynamics viscosity coefficient

$u_{\mathrm{i}}, u_{\mathrm{j}}(\mathrm{i}, \mathrm{j}=1,2,3)$ are the velocity components in $x, y$ and $z$ directions.

$p=$ static pressure, $\rho=$ density of fluid

At high breathing conditions, flow is turbulent. Hence realizable $k-\varepsilon$ turbulence model was selected to capture the airflow in the internal flow of the realistic human respiratory tract. Similar turbulence model was also selected by Green(2004) and Luo et al.(2004) in case of flow through human airways. The realizable $k-\varepsilon$ model provides good performance for the flow involving boundary layer under strong pressure gradient, flow separation and recirculation and was therefore adopted in the present study. Enhanced wall treatment in CFD solver was used to determine the flow parameters near the wall (Ansys-Fluent-14.5). Shear Stress Transport (SST) $k-\omega$ turbulence model for discrete phase modeling.

The transport equations for $k$ (turbulent kinetic energy) and $\varepsilon$ (turbulent dissipation) in the realizable $k-\varepsilon$ turbulent model are

$k$-equation:

$\frac{\partial}{\partial x_{j}}\left(\rho k u_{j}\right)=\frac{\partial}{\partial x_{j}}\left[\left(\mu+\frac{\mu_{t}}{\sigma_{k}}\right) \frac{\partial k}{\partial x_{j}}\right]+G_{k}-\rho \varepsilon$

$\varepsilon$-equation:

$$
\frac{\partial}{\partial x_{j}}\left(\rho \varepsilon u_{j}\right)=\frac{\partial}{\partial x_{j}}\left[\left(\mu+\frac{\mu_{t}}{\sigma_{\varepsilon}}\right) \frac{\partial \varepsilon}{\partial x_{j}}\right]+\rho C_{1} S \varepsilon-\rho C_{2} \frac{\varepsilon^{2}}{k+\sqrt{v \varepsilon}}
$$

where,

$C_{1}=\max \left[0.43, \frac{\eta}{\eta+5}\right]$ and $C_{2}$ and $\quad C_{2}=1.9$

$C_{\mu}=0.09$ are constants.

$\eta=S \frac{k}{\varepsilon}$

where, $S$ is the modulus of the mean rate of strain tensor, defined as 
$S=\sqrt{2 S_{i j} S_{i j}}$

$\mu$ is dynamic viscosity.

Turbulent/eddy viscosity is given as $\mu_{t}=\rho C_{\mu} \frac{k^{2}}{\varepsilon}$

Turbulent Prandtl number for k, $\sigma_{k}=1$,

Turbulent Prandtl number for $\varepsilon, \sigma_{\varepsilon}=1.2$

The shear stress transport (SST) $k-\omega$ turbulence model was used to study two-phase behavior. The transport equations for $k$ (turbulent kinetic energy) and $\omega$ (specific turbulent dissipation) in the $k-\omega$ turbulent model are:

$$
\begin{aligned}
& \frac{\partial}{\partial x_{i}}\left(\rho k u_{i}\right)=\frac{\partial}{\partial x_{j}}\left[\Gamma_{k} \frac{\partial k}{\partial x_{j}}\right]+G_{k}-Y_{k}+S_{k} \\
& \frac{\partial}{\partial x_{i}}\left(\rho \omega u_{i}\right)=\frac{\partial}{\partial x_{j}}\left[\Gamma_{\omega} \frac{\partial \omega}{\partial x_{j}}\right]+G_{\omega}-Y_{\omega}+D_{\omega}+S_{\omega}
\end{aligned}
$$

where,

$\mathrm{G}_{\mathrm{k}}$ and $\mathrm{G}_{\omega}$ represents the generation of turbulence kinetic energy and dissipation due to mean velocity gradients.

$\Gamma_{\mathrm{k}}$ and $\Gamma_{\omega}$ represent the effective diffusivity of $\mathrm{k}$ and $\omega$ respectively

$Y_{k}$ and $Y_{\omega}$ represent the dissipation of $k$ and $\omega$ due to turbulence.

\section{Equation of motion of particles:}

Particle Force Balance:

Discrete phase model (DPM) was used for the analysis of aerosol-particle motion in human airway. The force balance equation equates the particle inertia with forces acting on the particles and is written as:

$$
\frac{\partial u_{p}}{\partial t}=F_{D}\left(u-u_{p}\right)+\frac{g_{x}\left(\rho_{p}-\rho\right)}{\rho_{p}}
$$

First term in Eq. (7). represents drag force and second term is gravitational force.

The drag force per unit particle mass $\left(\mathrm{F}_{\mathrm{D}}\right)$ which is defined as:

$$
F_{D}=\frac{18 \mu}{\rho_{p} d_{p}^{2}} \frac{C_{D} \operatorname{Re}}{24}
$$

Where, $u$ is the fluid phase velocity, $u_{p}$ is the particle velocity, $\mu$ is the molecular velocity of fluid, $\rho$ is the fluid density, $\rho_{p}$ is the density of the particle, and $d_{p}$ is the particle diameter. Re is the relative Reynolds number, which is defined as:

$$
\operatorname{Re} \equiv \frac{\rho d_{p}\left|u_{p}-u\right|}{\mu}
$$

The drag coefficient $\left(\mathrm{C}_{\mathrm{D}}\right)$ formula can be calculated from

$$
C_{D}=a_{1}+\frac{a_{2}}{\operatorname{Re}}+\frac{a_{3}}{\operatorname{Re}^{2}}
$$

Where $a_{1}, a_{2}$ and $a_{3}$ are constants that apply to smooth spherical particles over several ranges of Re (AnsysFluent-14.5, User Guide).

\section{NUMERICAL SIMULATION}

A finite-volume based CFD software Ansys-Fluent-14.5 was used for numerical simulation of airflow and aerosol transport in realistic respiratory tract model (Patankar, 1980). The SIMPLE and SIMPLEC algorithm were used for the pressure-velocity coupling in air and discrete phase respectively (Patankar, 1980). The different terms of the transport equation were discretized using secondorder upwind numerical scheme for airflow. Since discrete phase model needs higher order discretization numerical schemes therefore QUICK scheme (Hayase, 1992) was used for momentum and turbulence equations. The wall $y+(Y$-plus) value which is non-dimensional is defined in terms of local Reynolds number(Re) which is used in CFD to recognize coarse or fine mesh near the wall(Salim and Cheah, 2009). The average $y+(Y-$ plus) values were approximately 2.5 throughout the wall. The convergence criteria was kept as $10^{-4}$ in line with Luo et al.(2004). The computational work was performed on an IBM workstation using Intel Xeon processor with 8 GB RAM and. A typical computation run time for air was approximately 8 hours for without tumor and 10 hours for with tumor. However, computational run time for air with aerosol was 15 hours.

\section{CFD VALIDATION}

The CFD code was validated against the data reported by Luo et al.(2004) for the non-tumor respiratory tract. In the present study, the maximum velocity in the respiratory tract was computed as $2.66 \mathrm{~m} / \mathrm{s}$ against the value $2.6 \mathrm{~m} / \mathrm{s}$ as reported by Luo et al.(2004), showing a deviation of $2.3 \%$, which is within acceptable limit. Average velocity in the tumorous respiratory model was also computed using two different CFD solvers 'AnsysCFX' and 'Ansys-Fluent'. The average velocity was computed at different cross sectional planes started from upstream to the downstream of tumor, which is plotted in Fig.5.

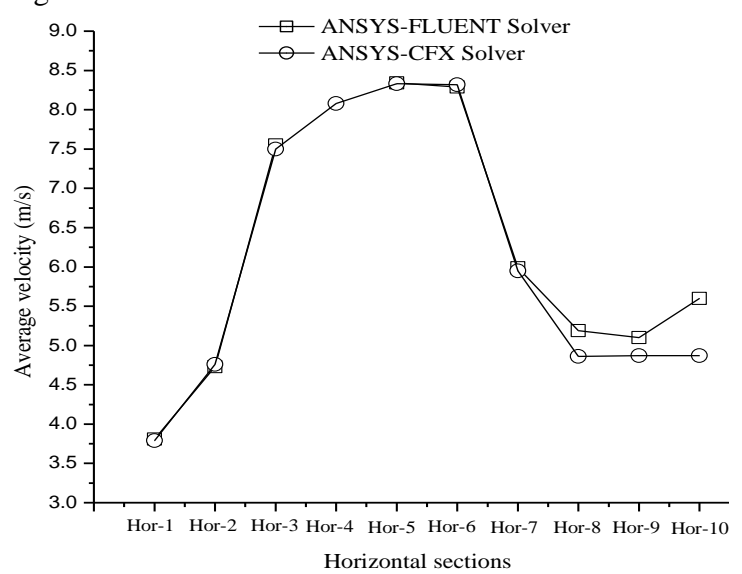

Fig. 5. Average velocity computed from two CFD solvers. 


\section{RESULTS AND DISCUSSION}

In this study, effects of tumor present in the respiratory tract on the flow pattern are investigated using three flow parameters, i.e., velocity, wall shear stress and deposition efficiency.

\subsection{Velocity Contours and Vectors}

\subsection{1: Normal Breathing Condition (6 litre/min)}

The tumor situated at the posterior wall of the trachea acts as obstruction in the respiratory tract and decreases the cross-sectional area, leading to an increase in flow velocity. The velocity contours at upstream and downstream region of the tumor are depicted through different vertical sections of airway in Fig.6a and $6 b$. These perpendicular cross sections as shown in Ver-1 to 4 (Fig.6a and 6b) are extended from posterior wall (having tumor) towards the anterior wall of trachea. Maximum velocity is obtained at the downstream of the tumor. The velocity contours show that obstruction in the airway path changes the airflow patterns.
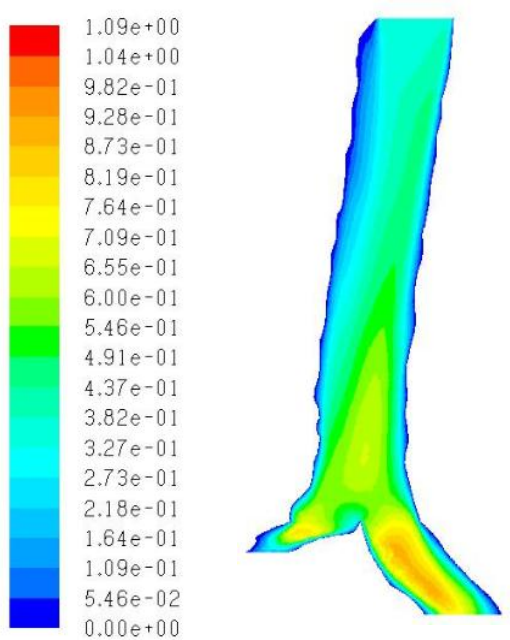

Ver-1

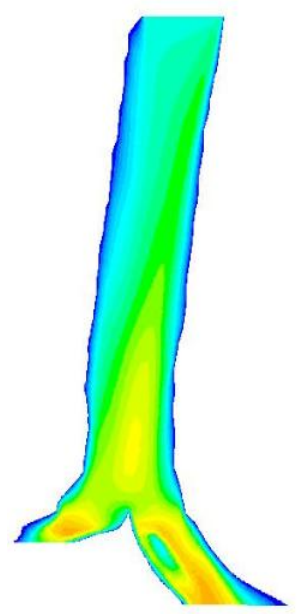

Ver-2

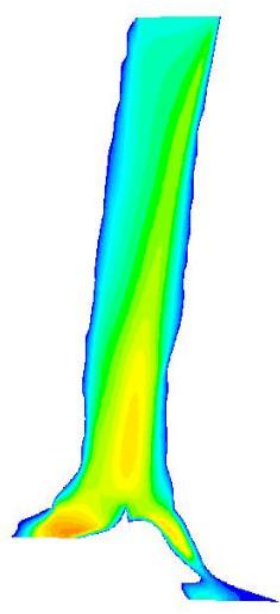

Ver-3

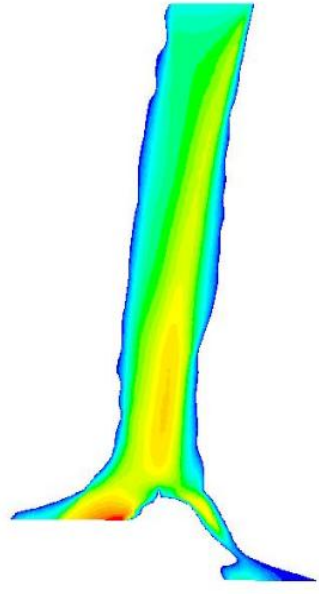

Ver-4

Fig. 6a. Velocity contours $(\mathrm{m} / \mathrm{s})$ in vertical sections of respiratory tract without tumor $(6$ litre/min)
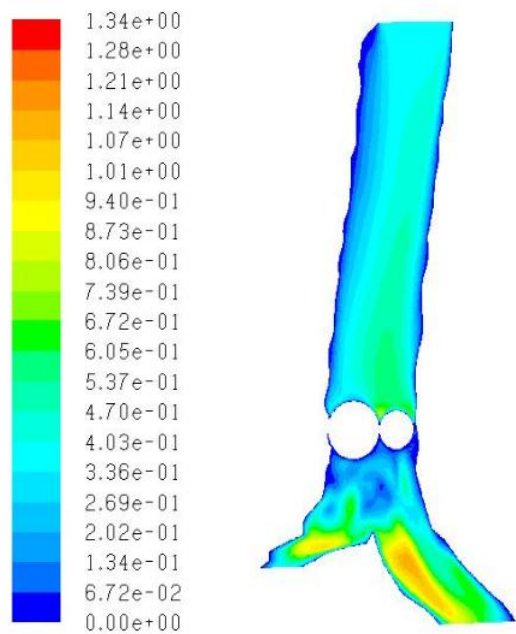

Ver-1

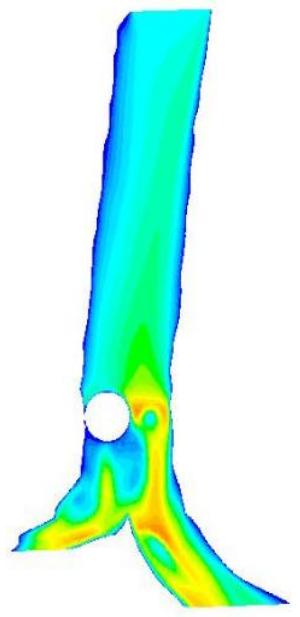

Ver-2

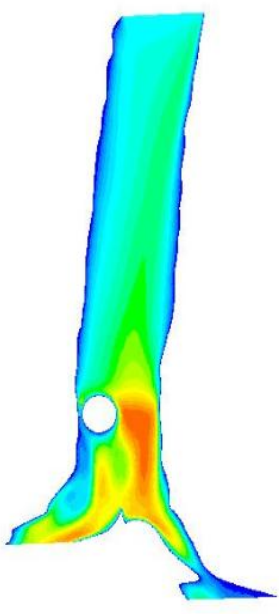

Ver-3

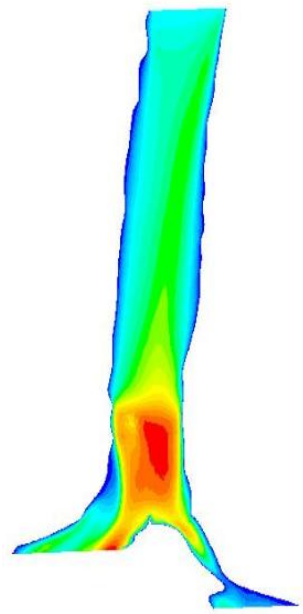

Ver-4

Fig. 6b. Velocity contours $(\mathrm{m} / \mathrm{s})$ in vertical sections of respiratory tract with tumor (6 litre/min)

The current numerical simulation shows a velocity contours upstream and downstream of the tumor in Fig.7. The velocity contours at upstream (Hor-1, Fig.7) is less affected by the presence of tumor. However, as the airflow moves towards the tumors (Hor-2 to Hor-7 in Fig.7), velocity increases rapidly due to reduced cross- sectional area. The airflow patterns have prominent effects on the downstream (Hor-8 in Fig.7) as compared to the upstream of the tumor. 


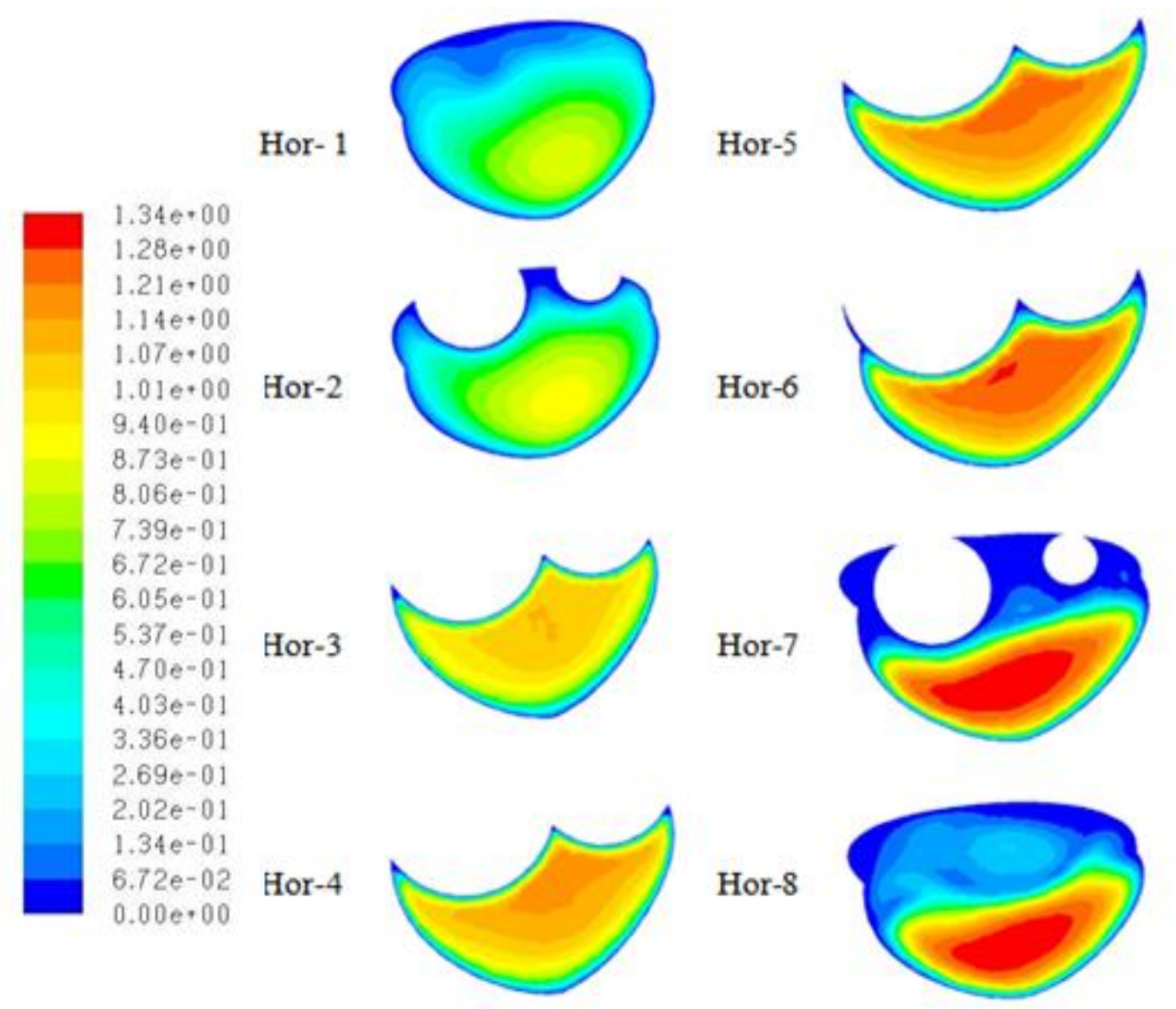

Fig. 7. Velocity contours $(\mathrm{m} / \mathrm{s})$ in horizontal cross-sections of respiratory tract with tumor

(6 litre/min)

The average velocity at different horizontal cross sections is shown in Fig.8. The maximum averaged velocities are obtained at the cross-sections Hor- 3, 4, 5 and 6 . The velocity ratio for with and without tumor is approximately 2.0 indicating that tumor blocks half of the airway of the respiratory tract.

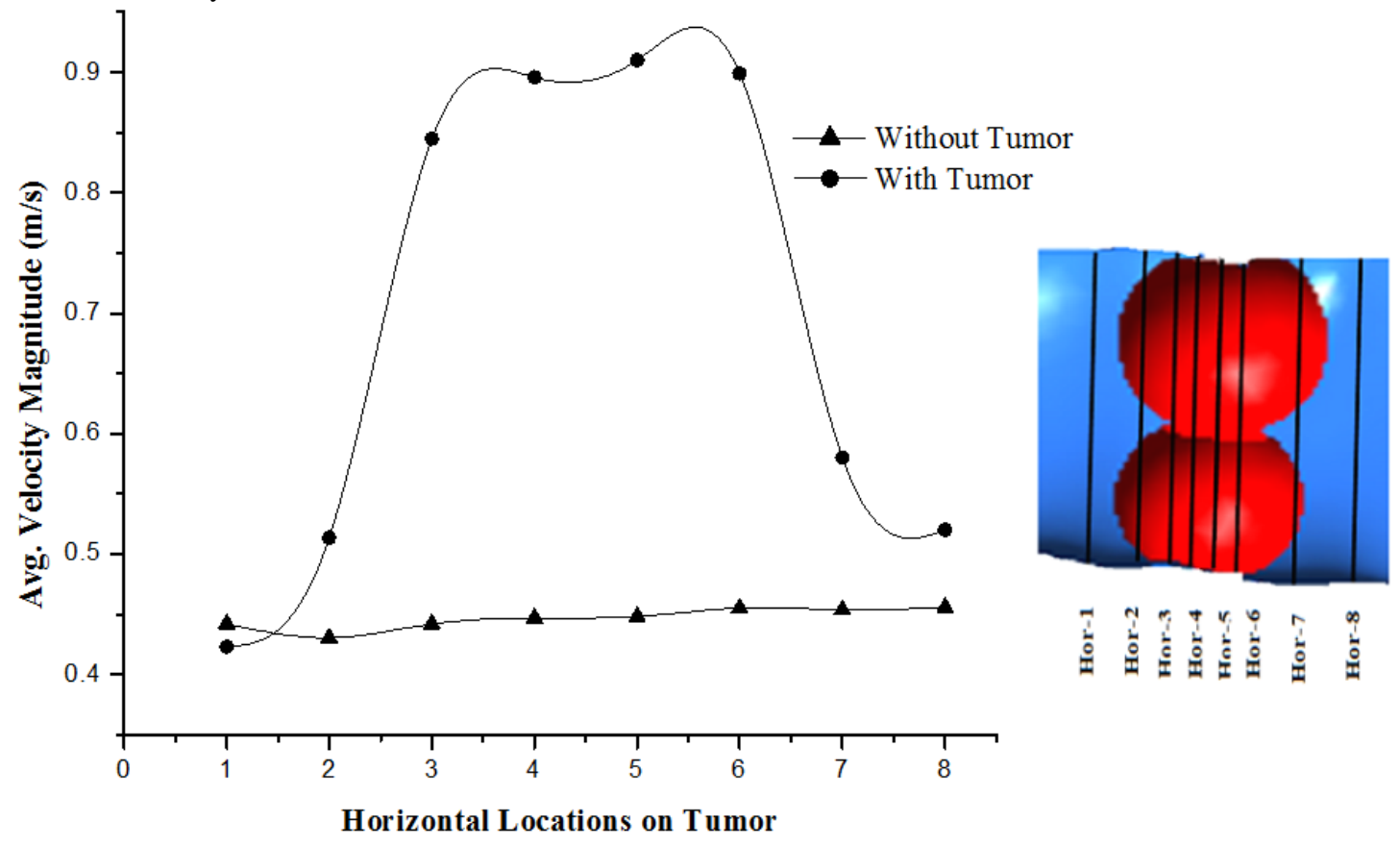

Fig. 8. Average velocity on tumor for normal breathing condition(6 litre/min) 


\subsection{2: Heavy Breathing Condition ( 45 litre/min)}

From the velocity contours shown in Fig.9a and 9b, it is noted that the velocity pattern in the upstream is almost same for both the cases (with and without tumor), but it changes rapidly in the downstream. Maximum air velocity is obtained near the anterior wall of trachea around the tumor (Ver-2 to 4 in Fig.9a) than near the posterior wall where tumor exists (Ver-1, in Fig.9b).

The cross section in Fig.9b (Ver-4) shows that high velocity extends up to the vicinity of the wall which may cause wall injury at this location.
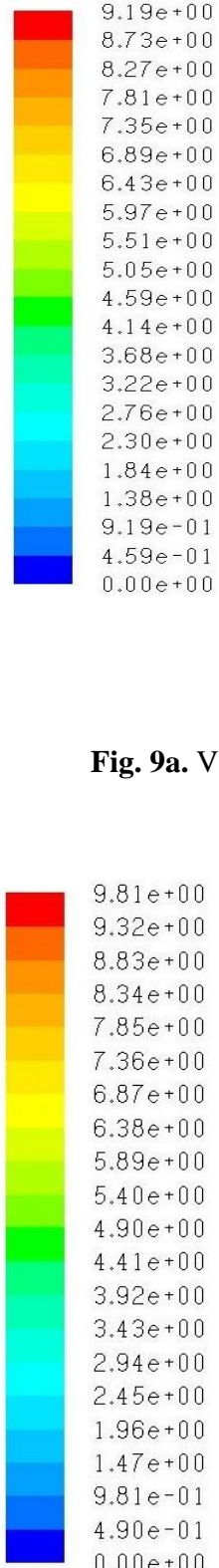

$8.83 e+00$

$8.34 \mathrm{e}+00$

$36 e+00$

$6.87 e+00$

$500+00$

$4.90 e+00$

$.43 e+00$

$.94 e+00$

$2.45 e+00$

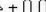

$0.00 e+00$

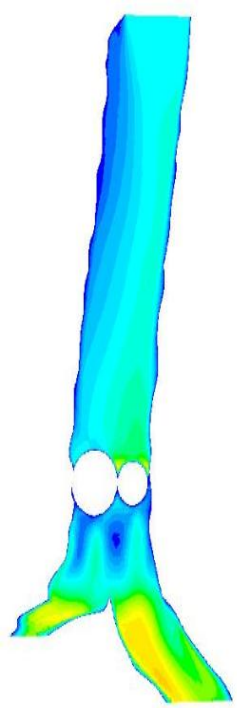

Ver-1

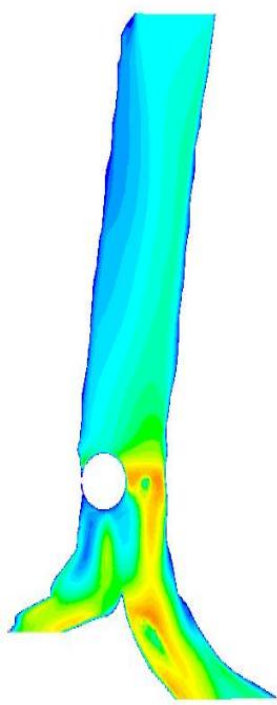

Ver-2

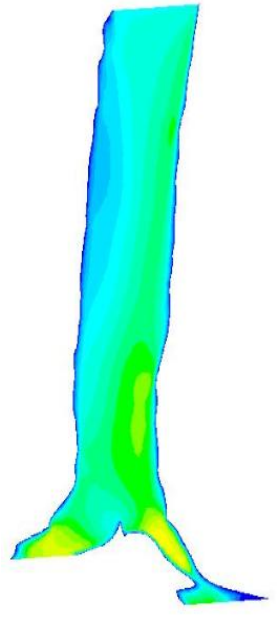

Ver-3

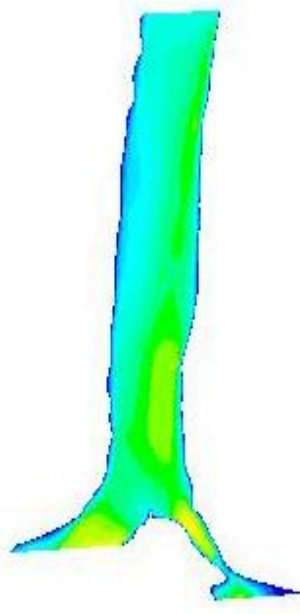

Ver-4

Ver-1

Ver-2

without tumor (45 litre/min) 


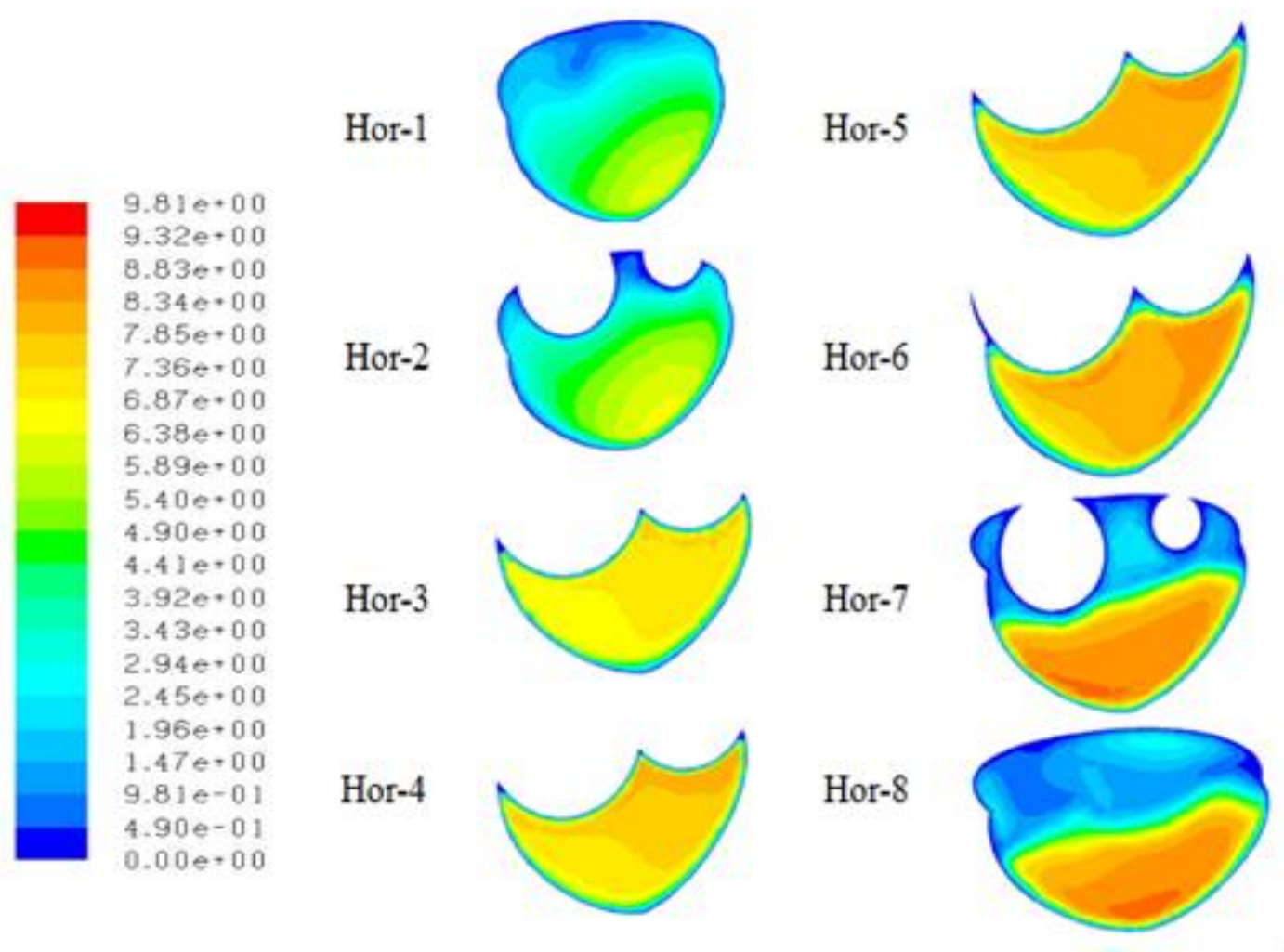

Fig. 10. Velocity contours $(\mathrm{m} / \mathrm{s})$ along the vertical cross-section of respiratory tract with tumor

Velocity recirculation zones are formed in the downstream region (Hor-9 \& Hor-10) of the airway as shown in Fig.11. A pair of counter-rotating vortices is generated at the cross-section of the anterior wall in the downstream region of the tumor due to the presence of obstruction (tumor) in the respiratory tract, which is similar to the wakes created the downstream for a flow past an object.
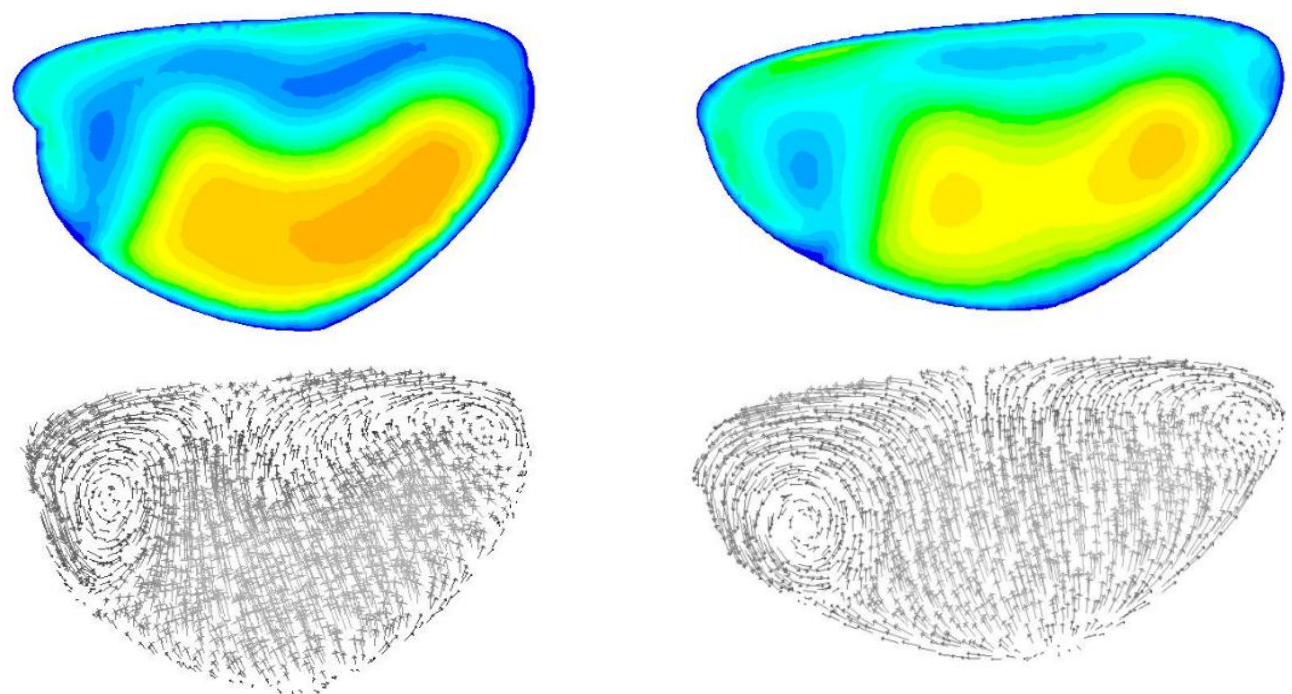

Hor-9

Hor-10

Fig. 11. Velocity contours $(\mathrm{m} / \mathrm{s})$ and velocity vector plots of the cross-section Hor-9 \& Hor-10 in the downstream of the tumor

Average velocity at different cross sections (Hor-1 to 8) for two cases (with and without tumor) is shown in Fig.12. The maximum ratio of average velocity with tumor to that of without tumor is 2.11 at Hor-5. This shows that the tumor blocks a little over half the crosssection area of the airway. 


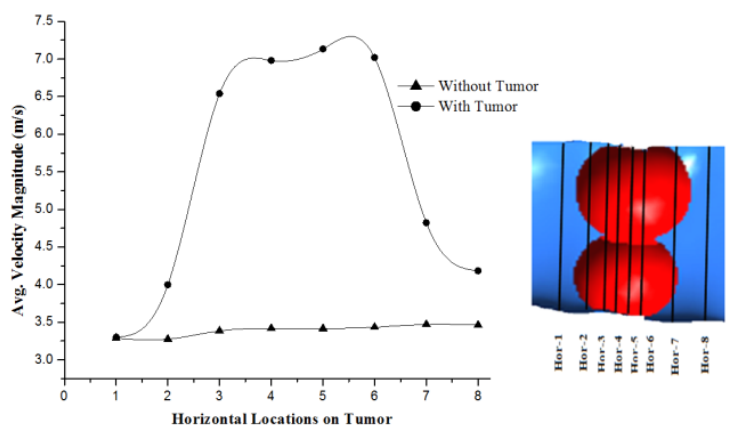

Fig. 12. Average velocity $(\mathrm{m} / \mathrm{s})$ on tumor for heavy breathing condition (45 litre/min)

\subsection{Wall Shear Stress}

\subsubsection{Normal Breathing Condition (6 litre/min)}

The variation of maximum WSS on wall around the tumor for the normal breathing condition is shown in Fig. 13. The peak wall shear stress value obtained at the horizontal location-7 is $0.12 \mathrm{~N} / \mathrm{m} 2$. Since the WSS is not more than critical value of $0.4 \mathrm{~N} / \mathrm{m} 2$. This indicates that there has no wall injury during the normal breathing condition.
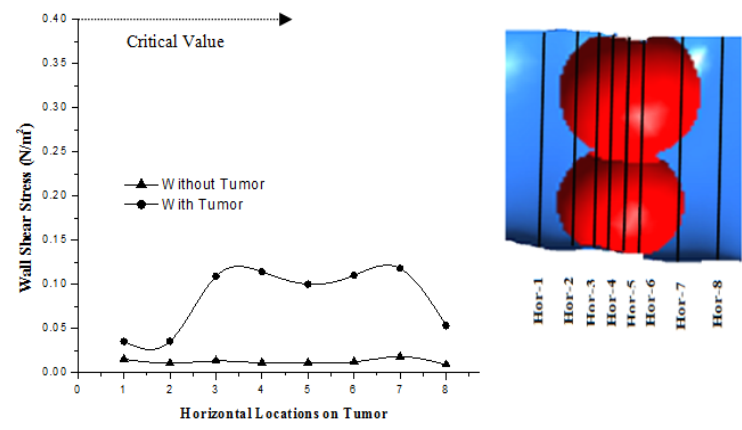

Fig. 13. Peak wall shear stress on tumor for normal breathing condition (6 litre/min)

\subsubsection{Heavy Breathing Condition (45 litre/min)}

The maximum wall shear stress for the two cases (with the tumor and without tumor) at different horizontal cross sectional planes (Hor-1 to 8) under heavy breathing condition is shown in Fig.14. The peak value of average wall shear stress in the presence of the tumor is about 8 times that of in the absence of the tumor. This high magnitude of shear stress may cause wear of epithelial layer/tissues.

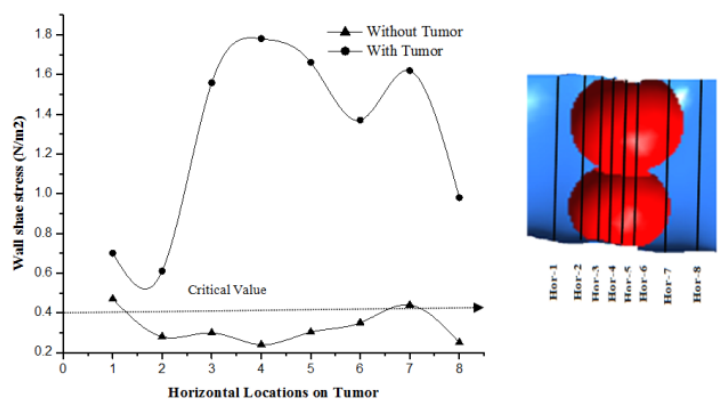

Fig. 14. Peak wall shear stress for heavy breathing condition (45 litre/min)
Due to the high velocity gradient near the surface of the airway and the presence of tumor in the respiratory tract, the wall shear stress increases very rapidly. Therefore, positions of cross sections-2 and 7 (located on tumor in Fig.14) are considered the most vulnerable location for the wall injury during the heavy breathing condition.

The peak value of wall shear stress at some locations of the interior walls of the human respiratory tracts is indicative of wall damage/injury, if this condition persists for long. Based on this information (called 'prognosis'), the pulmonologists can assess through medical imaging or using some other diagnostic techniques (called 'diagnosis') the extent of the wall injury actually occur at these locations and take remedial/corrective measures (called 'treatment').

\subsection{Effects of Particle Size and Inhalation Flow Rate on Drug Deposition}

Four different aerosol-particles (of size 1, 5, 7.5 and 10micron) are injected at the inlet of the trachea in order to supply maximum drug on the tumor location. These aerosol-particles are injected for different inspiratory flow rates (20, 45 and 60 litre/min). Higher inspiratory flow rates give inertial forces so that aerosol drugs can move towards tumor site. The deposition of drugs not only depends on aerosol size and inspiratory flow but also depends on tumor size. Tumors in respiratory tract create obstruction to the airflow. So if tumor size increases, drug deposition efficiency will also increase. Since aerosols are transported by airstream, therefore inspiratory flow rate plays an important role in the deposition efficiency of drugs.

The deposition of aerosol-drugs on tumor for different particle size and flow rate is given Fig.15. It is found low drug deposition on the tumor at low flow rate (20 litre/min) because particles do not obtain sufficient inertial forces to move. However, Deposition efficiency increases with the increase of particle size and inhalation flow rate. It is pertinent to mention that in case of turbulent flow regimes, minimum drug deposition efficiency on tumor is recorded with particle size of 5 micron. The deposition efficiency on tumor can however be improved with the increase of particle size. This indicates that the composition in an aerosol treating a lung tumor should contain particle size above 5 micron.

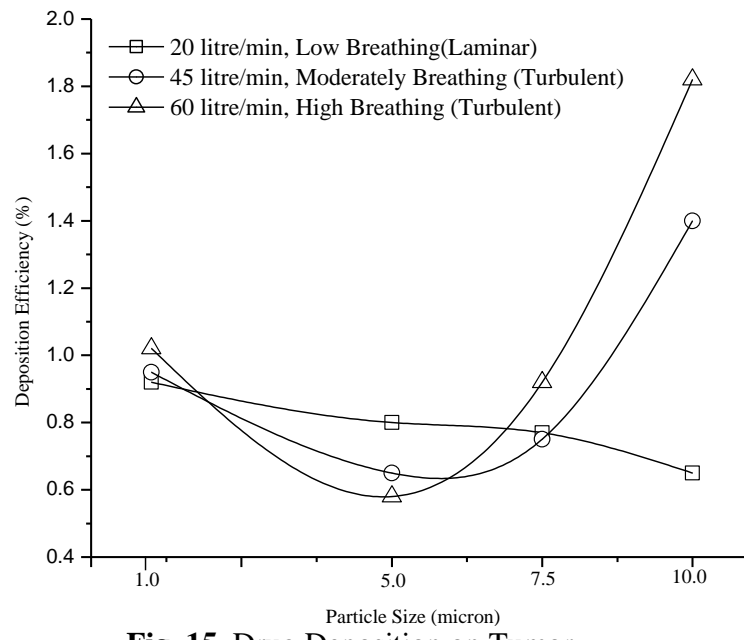

Fig. 15. Drug Deposition on Tumor 


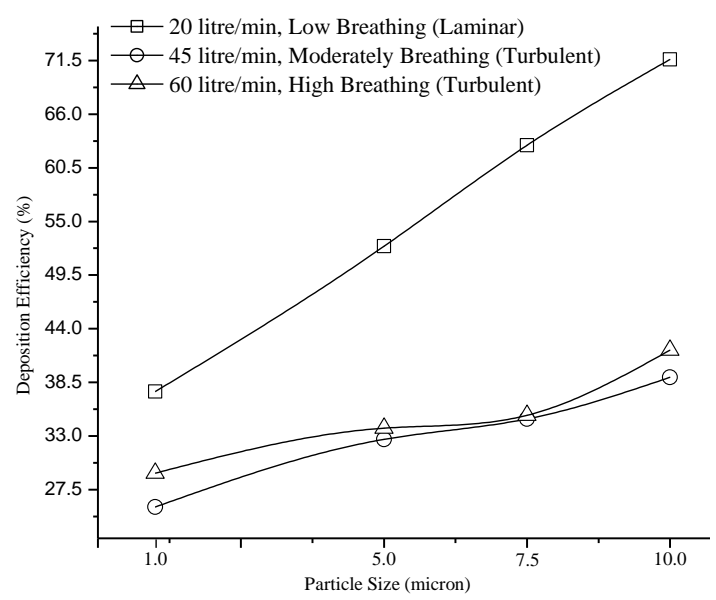

Fig. 16. Drug Deposition on Trachea

It is obtained that for moderate (45 litre/min) and higher breathing conditions (60 litre/min) aerosol deposition efficiency increases with the increase of particle size. Therefore, during the drug inhalation, patient is advised to inhale air rapidly so that the drug reaches to the location of tumor. Thus, patient skill is one of the most important factors that play crucial role during drug inhalation through inhaler.

Optimum drug must be delivered on the tumor to avoid the side-effects of drug. As the glomus tumor is found in the trachea, deposition efficiency of drugs in trachea was also calculated, which is shown in Fig.16. At low flow rate(20 litre/min) maximum drugs are deposited at the wall of trachea. While at higher flow rates $(45$ and 60 litre/min), minimum drugs are trapped in the trachea and hence deposited maximum on the tumor.

\section{CONCLUSIONS}

Inspiratory airflow in the rigid realistic human respiratory tract with and without tumor was numerically simulated for normal and heavy breathing conditions. Velocity contours and wall shear stress at different horizontal and vertical cross-sections were compared for both the cases. The findings from the study can be summarized as follows:

- Velocity contours show that the presence of tumor in the respiratory tract changes the flow pattern at the downstream of the tumor.

- Because of the presence of tumor in the respiratory tract, wall shear stress increases very rapidly due to the high velocity gradient.

- Wall shear stress values during the heavy breathing condition (45 litre/min) confirmed wall injury on the anterior wall of the tumor.

- The findings help in identifying the probable location of damage at the epithelial layer leading to injury to the airway. The magnitude of wall shear stress may serve as guideline to quantify the extent of damage possible.

- Aerosol size, inhalation flow rate and patient skill are important factors that play an important role during the drug delivery. It is found that in case of turbulent flow, the composition in an aerosol treating a lung tumor should contain particle size above 5 micron in order to increase drug deposition efficiency on tumor.

- Measurement of flow parameters, such as velocity, wall shear stress etc. in case of in-vivo condition always remains risky and therefore, the clinical data was not readily available with the authors. However, the outcome of this computational study can be corroborated with the experience of pulmonologists. Finally, the study will be useful for improving the drug delivery system through inhaler if the study can be repeated with the increasing size of the tumor; the results may also help in prognosis of the disease.

\section{ACKNOWLEDGEMENTS}

The authors are grateful to the department of science and technology, Government of India for providing financial support through its DST-FIST scheme to develop computational facility, which was used extensively to carry out the present study.

\section{REFERENCES}

Ansys-Fluent. Version 14.5. User Guide, Ansys Inc., USA. 2012.

Colaut F., Toniolo L., Scapinello A. and Pozzobon M. (2008). Tracheal Glomus Tumor Successfully Resected With Rigid Bronchoscopy: A Case Report, Journal of Thoracic Oncology, 3, 1065-1067.

Case Report: www.medicinenet.com/lung_cancer.

Green A. S.(2004). Modelling of peak-flow wall shear stress in major airways of the lung" Journal of Biomechanics, 37, 661-667.

Hayase T., Humphrey J.A.C. and Greif R.A. (1992). A consistently formulated QUICK scheme for fast and stable convergence using finite-volume iterative calculation procedures, J. Comput. Phys. 98, 108118.

Huang and Zhang. (2011). Micro-particle deposition and lobar distribution of mass flow in human upper respiratory tract model. Chinese Science Bulletin, 56, 380-385.

Kleinstreuer C. and Zhang Z. (2003). Targeted Drug Aerosol Deposition Analysis for a Four-Generation Lung Airway Model with Hemispherical Tumors. Journal of Biomechanical Engineering. 125, 197206.

Koombua K. and Pidaparti R.M. (2008). Inhalation induced stresses and flow characteristics in human airways through fluid-structure interaction analysis. Modelling and simulation engineering, vol 2008, article ID 358748 .

Lin C.L., Tawhai M.H., Geoffrey M., Hoffman E.A. (2007). Characteristics of the turbulent laryngeal jet and its effect on airflow in the human intra-thoracic airways. Respir Physiol Neurobiology, 157, 295-309.

Luo H.Y., Liu Y., Yang X.L. (2007) Particle deposition in obstructed airways. Journal of Biomechanics, 40, 3096-3104. 
Luo X.Y., Hinton J.S, Liew T.T., Tan K.K. (2004). LES modelling of flow in a simple airway model. Medical Engineering \& Physics, 26, 403-413.

Martonenan T.B. and Guan X. (2001). Effects of Tumors on Inhaled Pharmacologic Drugs. I. Flow Patterns. Cell Biochemistry and Biophysics, 35, 233-243.

Martonenan T.B. and Guan X.(2001). Effects of Tumors on Inhaled Pharmacologic Drugs. II. Particle Motion. Cell Biochemistry and Biophysics, 35, 245-253.

MIMICS 9.1, Materialise Inc., Belgium, NV.

Patankar, S.V.(1980). Numerical heat transfer and fluid flow. Hemisphere Pub. Corp., NY.

Parker K.L, Michael D.Z., Jessica S.D., Pratibha S.S. and Costas S.B.(2010). Tracheal Glomangioma in a Patient With Asthma and Chest Pain. Journal of Clinical Oncology, 28, e9-e10.

Salim M. S, and Cheah S.C. (2009). Wall y+ Strategy for Dealing with Wall-bounded Turbulent Flows. Proceedings of the International Multi Conference of Engineers and Computer Scientists. IMECS-2009, Hong Kong, China, March.

Segal R.A., Guan X., Shearer M. and Martonen T.B. (2000). Mathematical Model of Airflow in the Lungs of Children. I: Effects of Tumor Sizes and Locations. Journal of Theoretical Medicine, 2,199-213.
Shang Y., Huang Y., Huang H., Chong B., Dong Y., Zhao L. and Qiang L. (2010). Removal of Glomus Tumor in the Lower Tracheal Segment with a Flexible Bronchoscope: Report of Two Cases. Internal Medicine. 49, 865-869.

Srivastav V.K. and Jain A. (2011). Wall Shear Stress Study of Airflow in Realistic Human Airways Model: A CFD Analysis. Advances in Modeling, Optimization and Computing (AMOC-2011), 362367, ISBN: 9788186224748, IIT Roorkee, India, December.

Sulaiman M.Z.B. (2008). Flow analysis of COPD effects in human airways. ME Thesis. Universiti Teknologi Malaysia.

Watanabe M., Keigo T., Katsuaki O., Teruhiro A., Susumu T., Hideyuki S., and Shinsuke A.(1998). Successful Resection of a Glomus Tumor Arising from the Lower Trachea: Report of a Case. Japanese Journal of Surgery. 28, 332-334.

Weibel, E.R. (1963). Morphometry of the Human Lung. New York: Academic.

Zoltan G. and Zhang P.J. (2010). Glomus Tumor. Archives of Pathology \& Laboratory Medicine. 132, 1448-1452. 